\section{Behavior and Education Theory}

\section{P101 Comparison of Food Sources of Energy Among Adults that Skip Versus Consume Breakfast}

Stephanie Fanelli, MS, RDN, Stephanie.Fanelli2@osumc. edu, The Ohio State University, College of Medicine, Division of Medical Dietetics and Health Sciences, Atwell Hall, 453 W 10th Ave, Columbus, OH, 43210; Christopher Taylor, PhD, LD, RDN, FAND, The Ohio State University, College of Medicine, Division of Medical Dietetics and Health Sciences

Background: Breakfast consumption is associated with lower rates of chronic disease and higher intakes of vitamins and minerals, including calcium, folic acid, and zinc. Skipping breakfast may further pose a threat to diet quality, which may result in less desirable food choices later in the day. Previously, breakfast consumption in children was examined, yet less is known regarding adults.

Objective: To examine the proportional contribution of food sources to energy intake for adults who skipped vs ate breakfast.

Study Design, Setting, Participants: This cross-sectional study used data from 31,514 adults, aged 18 years+, from the 2005-2016 National Health and Nutrition Examination Survey. Participants were categorized according to whether they skipped $(n=4,993)$ or ate $(n=26,521)$ breakfast on the recorded day of intake.

Measurable Outcome/Analysis: Dietary intakes were assessed by a 24-hour dietary recall and meal occasions were self-designated by participants. Food sources were categorized into What We Eat in America Food Categories. Sums of energy were aggregated and used to determine the proportions of energy from each food source for the total day as well as during breakfast and snacks.

Results: Adults who skipped breakfast consumed greater proportions of total energy from mixed dishes, sweetenedbeverages, alcoholic beverages, and sandwiches, and less energy from breads, plant-proteins, milk, fruit, and eggs than those who ate breakfast. Breakfast energy mainly came from breads, eggs, and milk. Snack energy came from sweetened-beverages, alcoholic beverages, sweets, and savory snack-foods for those who skipped breakfast. For adults who ate breakfast, it came from sweets, alcoholic beverages, and savory foods; however, they also consumed more plant-proteins and fruit than those who skipped breakfast.

Conclusions: Making 1 poor nutritional decision, such as skipping breakfast, likely leads to other poor nutritional decisions throughout the day. Educational efforts should address breakfast's role in promoting nutritional adequacy and improving overall diet quality.

Funding: American Dairy Association Mideast.

\section{P102 Development of a Stage of Change Algorithm for Environmentally-Conscious Protein Choices}

Beth Carlton, bacarlton@my.uri.edu, The University of Rhode Island, 45 Upper College Rd, Kingston, RI, 02881;
Samantha Pender, The University of Rhode Island; Janette Bedoyan, MS, RD, The University of Rhode Island; Jade McNamara, PhD, RD, The University of Maine; Geoffrey Greene, $P h D, L D N, R D$, The University of Rhode Island

Background: Stage of Change (SOC)-based interventions have been effective for behavior change but few have been developed to assess sustainable eating behavior. No algorithm assessing motivational readiness to make environmentally-conscious protein choices has been previously developed.

Objective: To develop a SOC algorithm for environmentally-conscious protein (ECP) choices.

Study Design, Setting, Participants: Development was initiated using a cross-sectional survey of participants $(n=400)$ enrolled in introductory classes. Participants ranged from 18-24 years old and received extra credit for completion. The ECP SOC algorithm was recoded for this study categorizing participants as pre- or post-action.

Measurable Outcome/Analysis: ECP groups were compared using chi-square and t-tests for animal protein (AP) consumption, attitude toward food and long-term health impact (LTH), green eating SOC (GE), attitude toward plant-based foods (PBF), and attitude toward the impact of meat on the environment (MOE).

Results: Participants were predominantly white (83\%), female $(80 \%)$ and averaged $19 \pm 1.2$ years. Most participants (70\%) were in pre-action stages for ECP. Fifty-seven percent in pre-action consumed AP 4-6 days a week vs 41\% of those in post-action $(P<.001)$. Those in pre-action were less likely to always choose foods for LTH (22\%) vs post-action $(51 \%)$ $(P<.001)$. Eighty-eight percent in pre-action for GE were also pre-action for ECP, while $47 \%$ of post-action for GE were post-action for ECP $(P<.001)$. Fifty-one percent in preaction believed in eating mostly PBF to sustain health vs $79 \%$ in post-action $(P<.001)$. Lastly, post-action were more likely to contemplate the impact of MOE every time they ate meat $(26 \%)$ vs pre-action $(10 \%)(P<0.001)$.

Conclusions: The ECP SOC algorithm successfully distinguished pre- and post-action stages by behavior and attitudes. The consistency of these results with previous research provides support for the continued development of the ECP SOC algorithm. Future research is needed to validate this algorithm and to determine if it can be used for interventions to facilitate behavior change.

Funding: None.

\section{P103 Employing Social Norms to Influence Healthier Food Choices}

Denise Farrell, BS, MSA, dnfrrell@memphis.edu, University of Memphis, College of Education, Ball Hall Rm 100, Memphis, TN, 38152; Leigh Williams, PhD, University of Memphis; Karen Kitchens, PhD, University of Memphis

Background: To build on prior laboratory studies related to injunctive food norms, the goal of this field-experiment was to explore factors that influence patrons' food choices in a university cafeteria.

Objective: To evaluate the influence of food messaging (ie, injunctive norms) at a designated food station in the

Continued on page 565 